\title{
Síntese e Aplicação do Palmitato de Sódio para a Remoção do Metal Pesado (Cromo)
}

\author{
Maílla Isle Lopes*(Graduada em Engenharia Química na Universidade Federal Rural do Semi-Árido- \\ UFERSA); \\ Andressa Germana de Souza (Graduada em Engenharia Química na Universidade Federal Rural do \\ Semi-Árido-UFERSA). \\ *Email: lila_isle@hotmail.com
}

\begin{abstract}
resumo:
Muito se tem estudado sobre as características das substâncias tensoativas e suas inúmeras aplicações, inclusive na extração de cromo nos efluentes. O presente trabalho tem por objetivo a síntese e aplicação do tensoativo palmitato de sódio para a remoção do cromo de uma solução. A metodologia baseou-se em três etapas: síntese do palmitato de sódio, elaboração dos diagramas de fase pseudoternário (para três fases orgânicas distintas - hidrocarbonetos) e finalmente o preparo da solução de cromo para a extração por microemulsão. Nos resultados, os diagramas foram apresentados e em seguida discutidos, principalmente com relação a contribuição dos n-alcanos, para o equilíbrio destes, verificando possíveis mudanças nas regiões de microemulsão. A extração do cromo não foi compatível com os resultados esperados, pois, não houve redução significativa e de forma visual do metal pesado da solução preparada. Fato este, argumentado pela falta de afinidade do agente extrator (tensoativo) para com as fases aquosas e a fase microemulsionada (Winsor II), ocorrendo enfim a ineficiência do processo de extração por microemulsão, para este caso específico.
\end{abstract}

palavras-chave:

meio ambiente; tensoativos; diagrama pseudoternário; microemulsão; extração.

Espaço reservado para organização do congresso. 


\section{I ntrodução}

Os resíduos industriais como os metais pesados, quando expostos diretamente ao meio ambiente causam poluição dos lençóis freáticos, agridem o solo acarretando danos à saúde humana. Em virtude do grande prejuízo ao homem e seu habitat, é necessário minimizar os efeitos desses metais. Para tal, utiliza-se de técnicas de extração dos metais pesados na natureza e nesse contexto as substâncias tensoativas surgem como alternativa para redução desses impactos.

Substâncias tensoativas são aquelas que possuem moléculas anfifílicas, isto é, possuem na sua estrutura, duas regiões de polaridade opostas: uma apolar (hidrofóbica) e outra polar (hidrofílica). Devido suas propriedades químicas amplamente versáteis, os tensoativos atuam como solubilizantes, detergentes, dispersantes ou agentes emulsificantes. Atualmente, detém um vasto campo de aplicação tecnológica como por exemplo: agente farmacêutico, cosméticos, plástico, inibidores de corrosão, indústria petroquímica, extração de metais, coletores de flotação, dentre outras aplicabilidades. (ROSSI et al, 2006).

Este trabalho tem por objetivo geral a síntese e aplicação da substância tensoativa palmitato de sódio para efetuar testes de remoção do metal pesado cromo de uma solução, através da extração por microemulsão, a construção dos diagramas de fases pseudoternário os quais implicarão no preparo de microemulsões, base para a realização da extração.

\section{Microemulsão}

De acordo com Ezrahi et al., (1999) apud Formariz et al. (2005) as microemulsões (ME) podem ser entendidas como a formação de microestruturas em soluções aquosas de tensoativos, sendo um fenômeno comum de auto-organização molecular para atingir a estabilidade termodinâmica. As moléculas de tensoativos frequentemente se auto-agregam na presença de água formando uma rica variedade de estruturas, quando são modificados os parâmetros de concentração de tensoativos, presença de sal ou a temperatura. Esses agregados tornam-se mais estruturados quando um óleo ou mesmo outros componentes, como outro tensoativo ou um álcool de cadeia média, é adicionado ao sistema tensoativo-água. 


\section{Regiões de Winsor}

As microemulsões podem permanecer em equilíbrio com uma fase oleosa e/ou aquosa em excesso, formando compostos multifásicos, que no estado de equilíbrio apresentam quatro configurações dependendo da temperatura salinidade e composição. (NÓBREGA, 2003). São as chamadas Regiões de Winsor:

Região de Winsor I

Nessa região observa-se o equilíbrio entre a fase de microemulsão e a fase oleosa em excesso, em que o posicionamento das fases é definido pela diferença de densidade. (LOPES, 2003).

Região de Winsor II

Ao contrário da região Winsor I, esta representa o equilíbrio entre a fase de microemulsão e uma fase aquosa em excesso. (LOPES, 2003).

Região de Winsor III

Esse sistema apresenta três fases, onde a fase microemulsionada encontra-se em equilíbrio com uma fase aquosa e uma orgânica. (LOPES, 2003).

Região de Winsor IV

É caracterizado pela existência apenas de uma fase microemulsionada, ou seja, um sistema monofásico. (LOPES, 2003).

\section{Extração por microemulsão}

A técnica da extração por microemulsão consiste na mistura da fase oleosa, da fase aquosa, tensoativo e do cotensoativo em proporções previamente definidas, favorecendo as regiões de Winsor II. O sistema é colocado sob contato, com ou sem agitação mecânica, por um determinado tempo e em seguida mantido em repouso para a separação das fases (microemulsão e fase aquosa). A fase aquosa é então coletada para a caracterização através do método da absorção atômica, onde as concentrações do metal pesado são definidas. (LUCENA NETO, 2005).

\section{Metodologia}

Na fase de síntese do tensoativo utilizou-se a reação balanceada que resultará na formação do palmitato de sódio empregado como agente extrator, conforme descrita abaixo: 


$$
\mathrm{C}_{16} \mathrm{H}_{32} \mathrm{O}_{2}+\mathrm{NaOH} \rightarrow \mathrm{C}_{16} \mathrm{H}_{31} \mathrm{O}_{2} \mathrm{Na}+\mathrm{H}_{2} \mathrm{O}
$$

Conforme Lucena Neto (2005) o método experimental para gerar os diagramas pseudoternário baseia-se em dois passos:

i) Preparo da solução titulante: titula-se com uma solução aquosa uma mistura de cotensoativo + tensoativo, a uma razão C/T fixa até o ponto de solubilidade. A partir deste ponto adicionase $10 \%$ de solução aquosa obtendo então a solução titulante, que é constituída apenas de uma fase.

ii) Determinação da região de microemulsão: titula-se com a solução titulante misturas de água ou (cotensoativo/tensoativo) com óleo em proporções variadas, até os pontos de formação ou desaparecimento das fases.

Na terceira e ultima etapa deste estudo preparou-se a solução contendo o metal pesado cromo, para em seguida ser extraído. O sistema ótimo para ocorrer a extração por microemulsão é caracterizado conforme Moura (1997) pela presença de uma fase microemulsionada em equilíbrio com a fase aquosa em excesso, ou seja, a região de Winsor II. Definidas as proporções mássicas de tensoativo, cotensoativo, óleo e a solução do metal, a mistura foi homogeneizada e deixada em repouso durante um determinado tempo a fim de verificar a região de W II, visualmente caracterizada pela separação das fases oleosa e aquosa, sendo esta última em excesso.

\section{Resultados e Discussões}

\subsection{Elaboração das curvas de equilíbrio}

Foram construídos três diagramas pseudoternário variando a fase orgânica (FO), com o aumento do tamanho da cadeia carbônica (decano, dodecano e tetradecano). Para cada diagrama foram feitos dez pontos, cada um com suas respectivas proporções mássicas de tensoativo (mt), cotensoativo (mc), água (ma) e óleo (mo) sendo esses dados apresentados graficamente abaixo, com ajuda do software Excel 2007. 


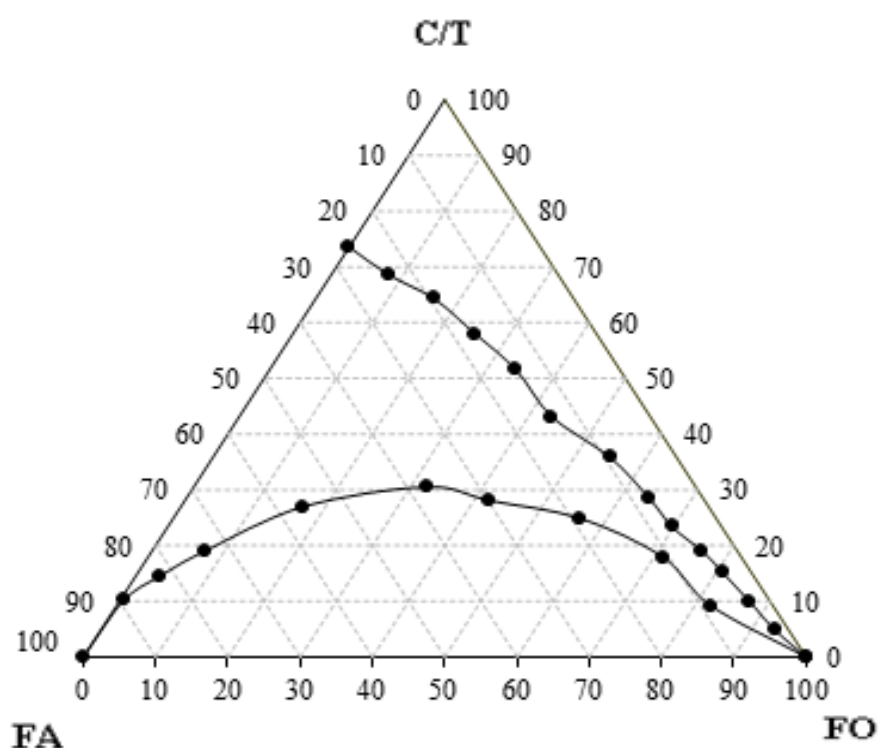

Gráfico I - Diagrama de fase pseudoternário para o decano $\left(\mathrm{C}_{10} \mathrm{H}_{22}\right)$

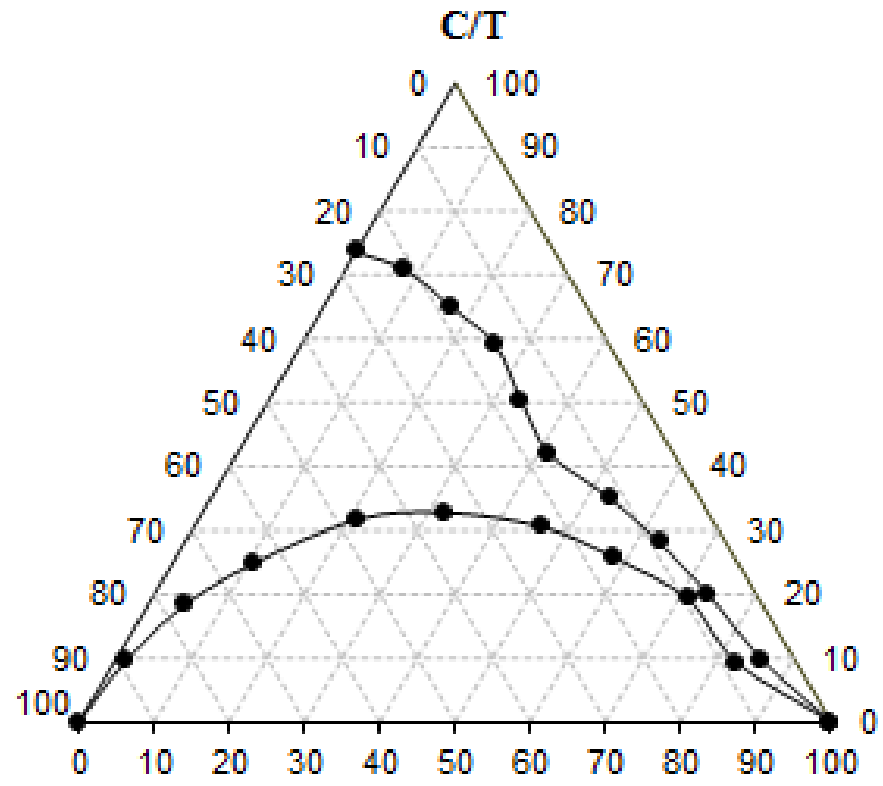

FA

FO

Gráfico II - Diagrama pseudoternário para o dodecano $\left(\mathrm{C}_{12} \mathrm{H}_{26}\right)$ 


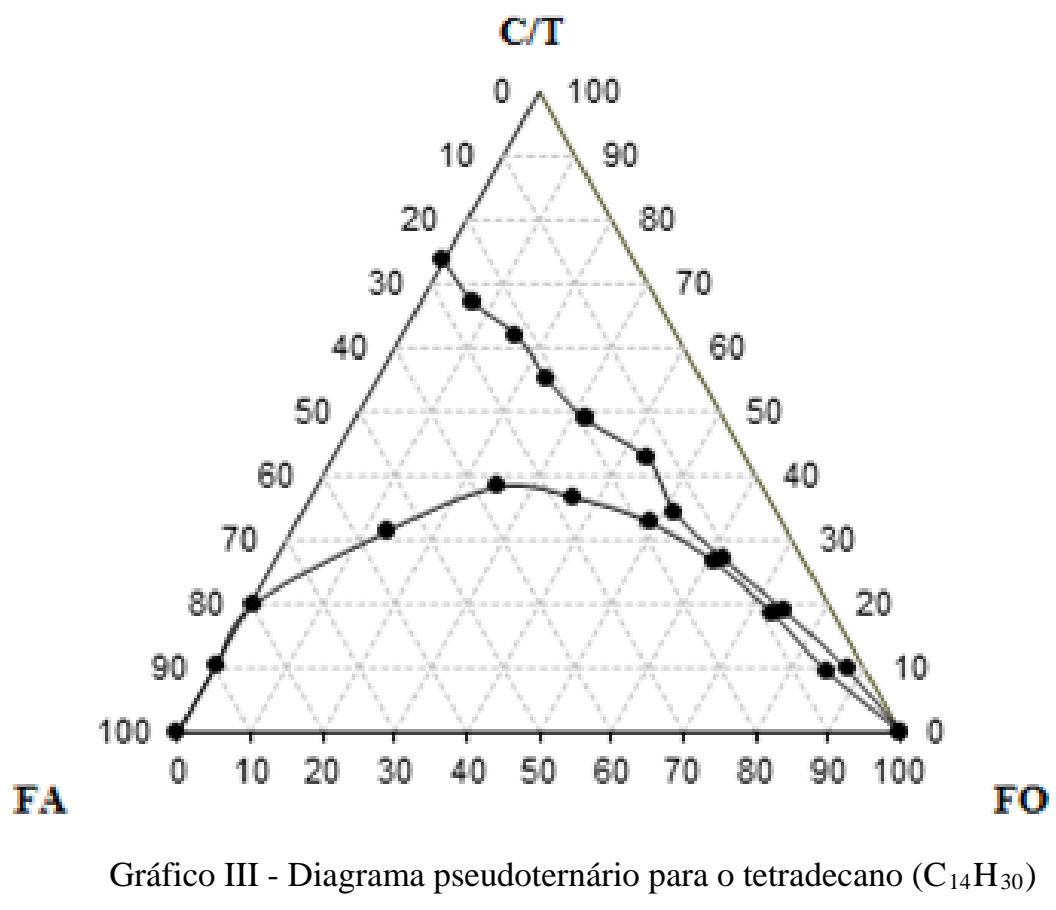

Considerando o parâmetro n-alcanos (hidrocarbonetos) utilizados, de acordo com os gráficos I, II e III constatou-se que o aumento do tamanho da cadeia carbônica favoreceu a redução das interações das microgotículas diminuindo o tamanho da região de microemulsão. Evidenciados por necessitar de uma menor quantidade de água para se atingir a região bifásica.

\subsection{Extração do cromo}

No presente estudo a migração do cromo para a fase microemulsionada não foi percebida visualmente. Dentre os fatores que podem ter influenciado a não realização da extração neste caso específico, destaca-se a escolha do agente extrator (tensoativo). Por apresentar uma cadeia muito longa $\left(\mathrm{C}_{16}\right)$, houve uma diminuição da solubilidade da microemulsão, não sendo observado a região de Winsor II, ocasionando a ineficiência do processo de extração.

\section{Conclusões}

A síntese do palmitato de sódio através da reação de saponificação ocorreu de maneira satisfatória o que permitiu produzir quantidade suficiente deste tensoativos para a realização desse estudo. 
Na elaboração dos diagramas pseudoternários o tamanho da cadeia carbônica dos hidrocarbonetos fase orgânica influenciam nas regiões de microemulsão. De forma que quanto maior a dimensão da cadeia, menor a solubilização da microemulsão. Desta forma, o decano apresentou maior região de microemulsão, seguido do dodecano e tetradecano.

A ineficiência do processo de remoção do cromo da solução através da extração por microemulsão pode ser atribuída ao fato do tensoativo palmitato de sódio, sintetizado em laboratório não apresentar características favoráveis a extração, entretanto estudos mais detalhados são necessários por se tratar de um estudo inédito, no que diz respeito a escolha do tensoativo.

\section{Synthesis and Sodium Palmitate Application For Heavy Metal Removal (chrome)}

Albstract: Much has been studied about the characteristics of the surface-active substances and their numerous applications, including the extraction of chrome in the effluent. This work aims at the synthesis and application of the surfactant sodium palmitate for chrome removal of a solution. The methodology was based on three steps: sodium palmitate synthesis, preparation of pseudoternary phase diagrams (for three separate organics - hydrocarbons) and finally the preparation of chrome solution for extraction by microemulsion. In the results, the diagrams were presented and discussed then, especially regarding the contribution of nalkanes, for the balance of these by checking possible changes in the regions of microemulsion. The chrome extraction was not compatible with the expected results because there was no significant reduction and visually heavy metal of the prepared solution. This fact, argued by the lack of affinity of extracting agent (surfactant) towards the aqueous phase and the microemulsion phase (Winsor II), finally going to inefficient extraction procedure microemulsion, for this particular case.

Keywords: environment; surfactants; pseudoternary diagram; microemulsion; extraction.

\section{Referências bibliográficas}

FORMARIZ, Thalita Pedroni. et al. Microemulsões e fases líquidas cristalinas como sistemas de liberação de fármacos, Revista Brasileira de Ciências Farmacêutica, V., 41, n. 3,: 2005.

LUCENA NETO, Marciano Henrique. Estudo da influência de tensoativos em sistemas microemulsionados na extração de gálio e alumínio. Tese Doutorado, Engenharia Química Universidade Federal do Rio Grande do Norte, 160 f: 2005. 
MOURA, Maria Carlenise Paiva de Alencar. Otimização do processo de recuperação do cromo de efluentes de curtumes por microemulsões em escala semi piloto. Dissertação Mestrado, Engenharia Química - Universidade Federal do Rio Grande do Norte, 137 f : 1997.

NÓBREGA, Geraldine Angélica Silva. Remoção da água do gás natural por absorção utilizando sistemas microemulsionados. Dissertação Mestrado, Engenharia Química Universidade Federal do Rio Grande do Norte, 121 f: 2003.

ROSSI, Cátia Guaraciara Fernandes Teixeira. et al. Tensoativos: uma abordagem básica e perspectivas para aplicabilidade industrial. Revista Universidade Rural, Seropédica, V., 25, n.12,: 2006. 